\title{
ANÁLISIS DE VIABILIDAD DE IMPLEMENTACIÓN DE LA TECNOLOGÍA WIBACK EN ZONAS RURALES COLOMBIANAS
}

\author{
Juan Garzón Álvarez \\ Universidad Católica de Oriente - GIMU
}

fgarzon@uco.edu.co

(Tipo de Artículo: Revisión. Recibido el 10/04/2014. Aprobado el 18/05/2014)

\begin{abstract}
RESUMEN
Resumen. En este artículo se presenta el análisis de la viabilidad de la implementación de la tecnología Wireless Backhaul (WiBack) como alternativa para la conexión a Internet en aquellos lugares que por su difícil acceso y/o ubicación, no es viable su interconexión a través de nodos de Fibra Óptica en Colombia. Se analizan aspectos técnicos y económicos teniendo en cuenta la situación colombiana; sin embargo, éste estudio puede ser aplicado igualmente a países con bajo índice de desarrollo o incluso en regiones rurales de difícil acceso en los países desarrollados.
\end{abstract}

\section{Palabras clave}

Configuración diferencial, modelado cinemático, Robótica móvil, trayectorias.

\section{ANALYSIS OF THE FEASIBILITY OF WIRELESS BACKHAUL TECHNOLOGY IMPLEMENTATION IN COLOMBIAN RURAL AREAS}

\author{
ABSTRACT \\ This article presents the analysis of the feasibility of the implementation of the Wireless Backhaul (WiBack) technology as an \\ alternative for Internet connection in Colombian places which, because of its location, it is not feasible a connection with optical \\ fiber nodes. Technical and economic aspects are analyzed taking into account the situation in Colombia; however, this study can \\ be applied in countries with low rates of development or even in difficult access rural areas in developed countries.
}

\section{Keywords}

Backhaul, ICT, IEEE 802.21, Mesh Networks, WiBack.

\section{ANALYSE DE VIABILITÉ DE I'IMPLÉMENTATION DE LA TECHNOLOGIE WIBACK DANS ZONES RURALES DE LA COLOMBIE}

\begin{abstract}
Résumé.
Cet article présente l'analyse de viabilité de l'implémentation de la technologie Wireless Backhaul (WiBack) comme une alternative pour se connecter sur Internet dans endroits qui présentent difficile accès ou emplacement, pour lesquels n'est pas viable une liaison en utilisant des nœuds de fibre optique dans la Colombie. On analyse les questions techniques et économiques en considérant la situation colombienne ; cependant, cet étude peut être appliqué aussi aux pays avec un bas niveau de développement ou pour régions de difficile accès dans pays développés.
\end{abstract}

Mots-clés.

Backhaul, IEEE 802.21, Reseaux maillés, TIC, WiBack. 


\section{INTRODUCCIÓN}

Cada vez se hace más evidente la importancia del uso de la red de Internet para aplicaciones que van desde el hogar hasta las altas esferas gubernamentales. La Internet se presenta ubicuamente en todos los aspectos de nuestra vida diaria y es por esto que se ha identificado como factor fundamental en el desarrollo de los países. Existen diversos estudios [1], [2], [3], que muestran como el aumento en la cobertura de Internet en un país aumenta proporcionalmente el PIB y disminuye la tasa de desempleo. Además, los empleos generados en la industria TIC, en contraste con empleos tradicionales en Colombia como los derivados del agro y la construcción entre otros, tienden a ser mejor remunerados y proporcionan mayor competitividad al país.

En vista de esto, el gobierno colombiano a través del ministerio de las Tecnologías de la Información y las Comunicaciones (MinTic) viene desarrollando un plan denominado "Vive Digital", con el cual busca ampliar la cobertura de Internet al $80 \%$ de los municipios del territorio nacional con nodos de Fibra Óptica (FO) [4]. Mediante este plan, el gobierno busca trazar la ruta que permita impulsar el salto tecnológico que potencie el desarrollo del país.

La pregunta es ahora, ¿qué pasa con aquellos lugares que debido a su difícil situación geográfica queden marginados de la cobertura de dicho plan? Es aquí donde a través de este análisis se busca revisar una posible alternativa de conexión a Internet que permitan ser integrada al plan Vive Digital y colaborar así con la disminución de la llamada Brecha Digital (término empleado para establecer la diferencia entre las personas o comunidades que tienen acceso a las diversas formas de TIC y aquellas que no) [5].

Después de un análisis previo que se ha realizado acerca de diferentes tecnologías potencialmente útiles, se ha decidido enfocar el estudio en la tecnología WiBack (Wireless Backhaul) la cual viene siendo desarrollada por el instituto alemán Fraunhaufer para aplicaciones en área rurales [6], [7].

\section{ANÁLISIS DEL ESCENARIO}

El acceso a la red de Internet ha dejado de ser un lujo o una curiosidad tecnológica para convertirse en una herramienta de vital importancia para el desarrollo de una región. Diversos estudios demuestran que el desarrollo de las tecnologías de la información y las comunicaciones (ámbito al cual pertenece la Internet) representa desarrollo económico y social, especialmente en países en desarrollo.

Un estudio realizado por Naciones Unidas concluye que la tasa de pobreza de los países disminuye a medida que aumenta el número de usuarios de Internet [8]. Otro caso demostrado por la Universidad de Columbia, sustenta que en Chile, un aumento de 10 puntos porcentuales en la penetración de Internet redujo el desempleo en un $2 \%$ [9]. Debido a la globalización que experimenta el planeta, la masificación de internet repercute positivamente en la competitividad de una nación.

Así pues, mediante el plan Vive Digital el ministerio TIC de Colombia pretende alcanzar una conexión a Internet del $50 \%$ de los hogares colombianos al finalizar el año 2014 .En el momento de lanzamiento del proyecto se tenía una participación del $27 \%$ y en el último reporte del DANE se presenta una conexión del $35,7 \%$ al finalizar el 2013 [10]; aunque la meta aún está lejos por cumplirse, se abona el gran progreso que se ha presentado.

Estas conexiones se vienen realizando principalmente mediante enlaces de FO a través del "Proyecto Nacional de Fibra Óptica". En este proyecto se estableció que al finalizar el cuatrienio presidencial, se debía tener cobertura con enlace de FO en las cabeceras municipales de 700 municipios colombianos. Al finalizar el año 2013, se contaba con la cobertura de 777 cabeceras municipales lo cual representa un avance del $115 \%$ frente a la meta propuesta y se espera que aumente aún más al finalizar el año 2014 [11].

Pero, ¿qué pasará con aquellos municipios a los cuales no llegará la FO? y ¿cómo ampliar la red más allá de las cabeceras municipales?

Hasta el momento la mejor solución ha sido llevar Internet a estos lugares por medio de la tecnología satelital, prueba de ello está la firma del convenio en el año 2012 entre la multinacional O3b Networks y Skynet Colombia [12] mediante el cual se pretende llevar Internet de alta velocidad y a bajo costo a regiones como la Amazonía colombiana, sin embargo, existen otras alternativas entre las cuales se encuentra WIBACK tecnología que será evaluada en el este artículo.

Por otro lado, paralelo al despliegue de las redes de datos se debe trabajar en la masificación del uso de las terminales que permitan la conectividad a la Internet tales como computadores, tabletas electrónicas, teléfonos inteligentes, entre otros, por parte de los ciudadanos y todo tipo de empresas.

Mediante un estudio complementario a las actividades del Plan Vive Digital, se han identificado dos grandes barreras que dificultan la masificación del uso de Internet relacionadas con el usuario [13]: como primera barrera se encontró que algunas personas $\mathrm{y} / \mathrm{o}$ empresas no ven la necesidad de su uso ya que le consideran poco útil. Como segunda barrera se encontraron aspectos económicos desfavorables de los ciudadanos y/o empresas ya que no solo se requiere del pago de servicio de la Internet sino que también como es lógico, se debe contar con una terminal que implica un costo adicional. 
Para tratar de subsanar esta última barrera, el gobierno nacional viene buscando la manera de proveer facilidades económicas para que las personas de más bajos recursos puedan adquirir terminales a precios más asequibles. Así pues, en el marco de las acciones del plan Vive Digital, el gobierno nacional eliminó los aranceles de importación para computadores, tabletas electrónicas, teléfonos inteligentes y repuestos relacionados a las terminales importación y el IVA para el servicio de internet de los estratos 1, 2 y 3 lo cual se traduce en que en Colombia se consigan los equipos de cómputo con los precios más bajos de la región tal como lo indica MinTlc en su portal web [14]. Con esto se ha logrado pasar de $38,5 \%$ de los hogares colombianos que cuentan con al menos un computador en el 2012 a 42,2\% en el 2013 [10].

Gran parte del territorio nacional está atravesado por tres cordilleras: Cordillera Central, Cordillera Oriental y Cordillera Occidental y en medio de estas cordilleras se encuentra cerca del $60 \%$ del total de la población [15].

Esto ocasiona que en muchas situaciones resulte sumamente complicado la conexión de algunas poblaciones por medio de enlaces de fibra óptica lo cual es la idea principal del gobierno nacional, es por esto que se debe recurrir a otras tecnologías que permitan extender las redes más allá de las cabeceras urbanas de manera rápida, efectiva y económica y es en este punto donde a partir de este análisis se pretende dar a conocer las diversas ventajas que podría conllevar la implementación de la tecnología Wireless Backhaul - WiBack.

\section{TECNOLOGÍA WIBACK}

Wireless Backhaul -WiBack- es una red de acceso inalámbrico a Internet que usa tecnologías existentes para construir una red de conexión de radio de largo alcance a través de enrutadores WiBack. Esta tecnología viene siendo desarrollada por el Instituto alemán Fraunhofer a través del proyecto denominado Focus [16].

Entre las principales características de esta tecnología, se encuentra su reducido costo tanto de adquisición como de mantenimiento, su propiedad de autoconfiguración y su gran resistencia [17].

Por medio de esta tecnología, una red de Internet puede ser expandida por cientos de Kilómetros, además, si uno de los enrutadores fallara, la red se restaura así misma de tal manera que el enlace no se cae [18]. Los enrutadores WiBack pueden ser instalados en construcciones existentes de gran altura, torres de agua o similares como se muestra en la Fig. 1. Cuentan además con interfaces GSM y UMTS por lo tanto, esta red tiene la capacidad de soportar telefonía móvil [19].

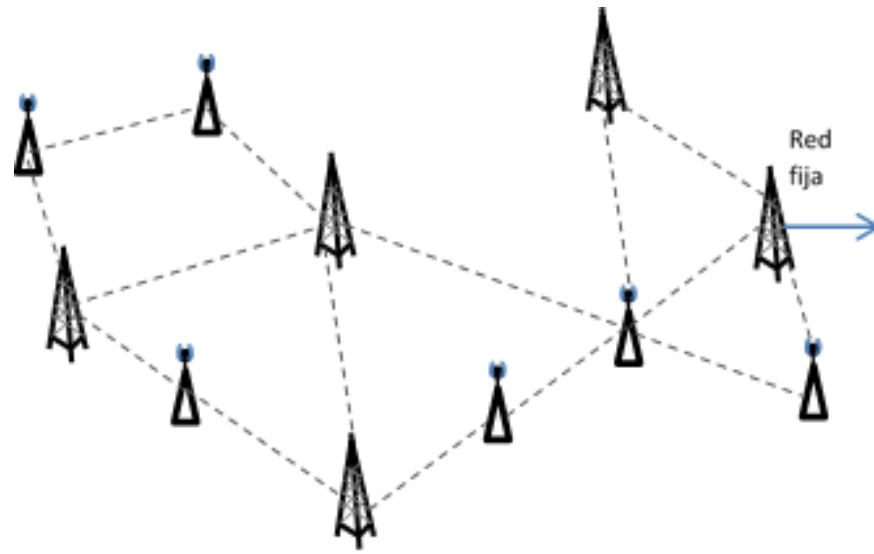

Fig. 1. Red WiBack

Otra gran ventaja es su bajo consumo de energía (unos $6 \mathrm{~W}$ ) por lo que pueden ser alimentados por medio de paneles solares [20]. Otras aplicaciones de esta tecnología se encuentran en los eventos de participación masiva, permitiendo el aumento de la capacidad de la red existente y/o como soporte en telecomunicaciones de emergencia en lugares que hayan sufrido algún tipo de catástrofe.

Es una tecnología Carrier Grade (extremadamente confiable), la cual garantiza una disponibilidad de por lo menos cinco nueves [21].

La arquitectura WiBack mostrada en la figura 2, surgió como inspiración de los resultados del proyecto de la Unión Europea "CARMEN" el cual lidera uno de los cambios fundamentales del futuro de la Internet: proveer soluciones de retorno para redes de acceso de radio [22], [23]. La principal novedad de este proyecto es la implementación de redes en Malla, lo que asegura una solución efectiva, flexible, de alta confiabilidad y principalmente, a muy bajo costo.

Al igual que en el proyecto CARMEN, el plano de control en WiBack se lleva a cabo a partir del estándar IEEE 802.21 el cual permite el traspaso perfecto entre tecnologías heterogéneas [24]. El componente principal del plano de control es la IMF (Interface Management Function) [25] el cual extiende las funcionalidades del estándar 802.21 [26].

Para administrar una red WiBack la Función de Gestión de Topología (Topology Management Function, TMF) es ejecutada como un proceso continuo el cual sigue a un enfoque de gestión maestro/esclavo y está diseñado para permitir la preferencia de ciertas tecnologías en áreas geográficas específicas o bandas de frecuencias [27]. 


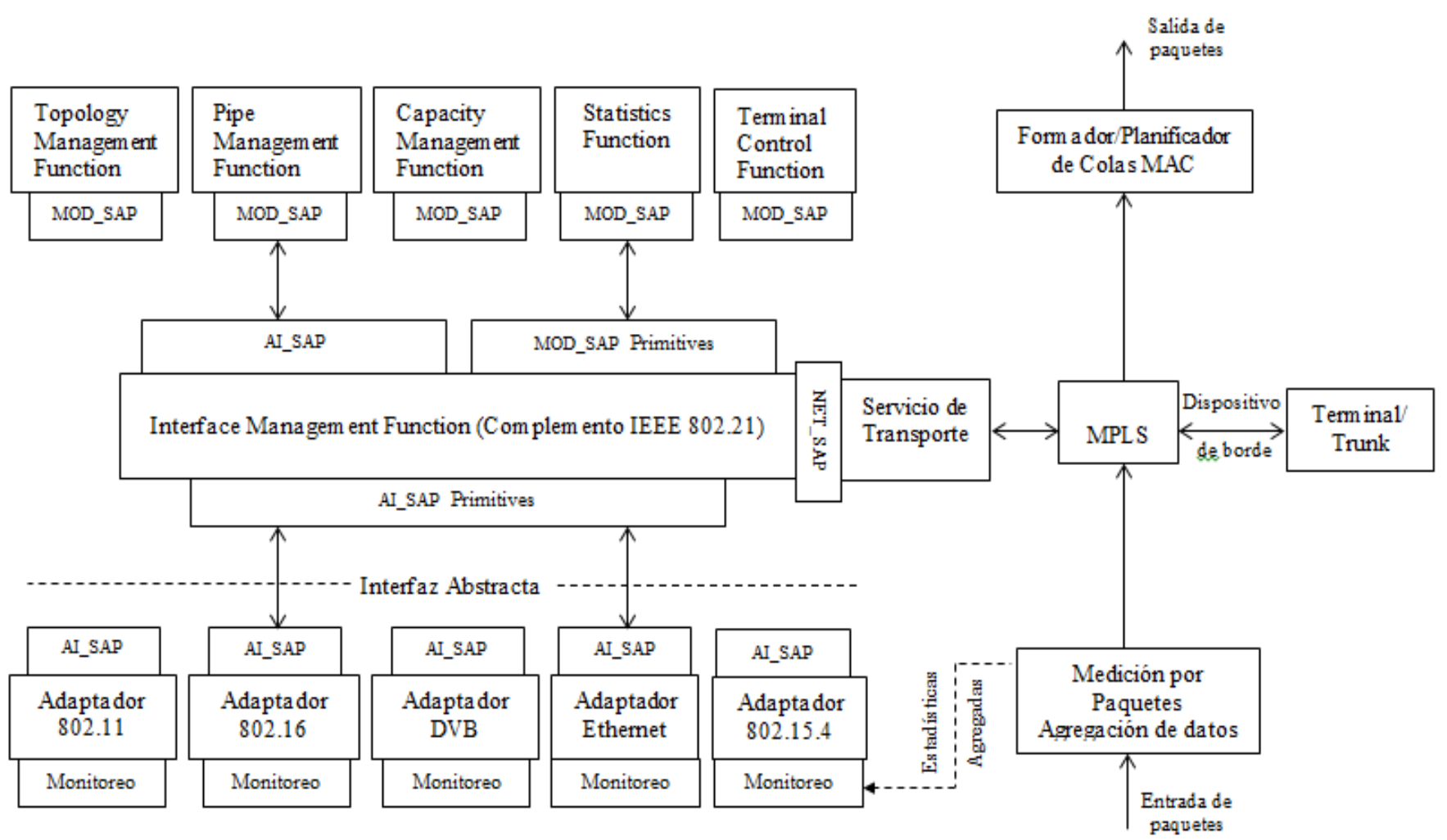

Fig. 2. Plano de Control y Plano de Datos

\section{CARACTERISTIICAS DE LAS REDES EN MALLA}

Es una topología de red en la cual todos los nodos están conectados entre sí, por lo tanto, entre cada par de nodos existen varios caminos, de tal manera que si uno falla la comunicación se efectúa por uno de los caminos alternativos [28], [29].

Como se puede apreciar en la Fig. 3, no se requiere de servidor ni de nodo central con lo que se reduce los costos por mantenimiento. Una de las principales ventajas de las redes en malla es que son autoconfigurables [30], así, si un nodo falla el mensaje toma una nueva ruta y el enlace no se interrumpe [31].

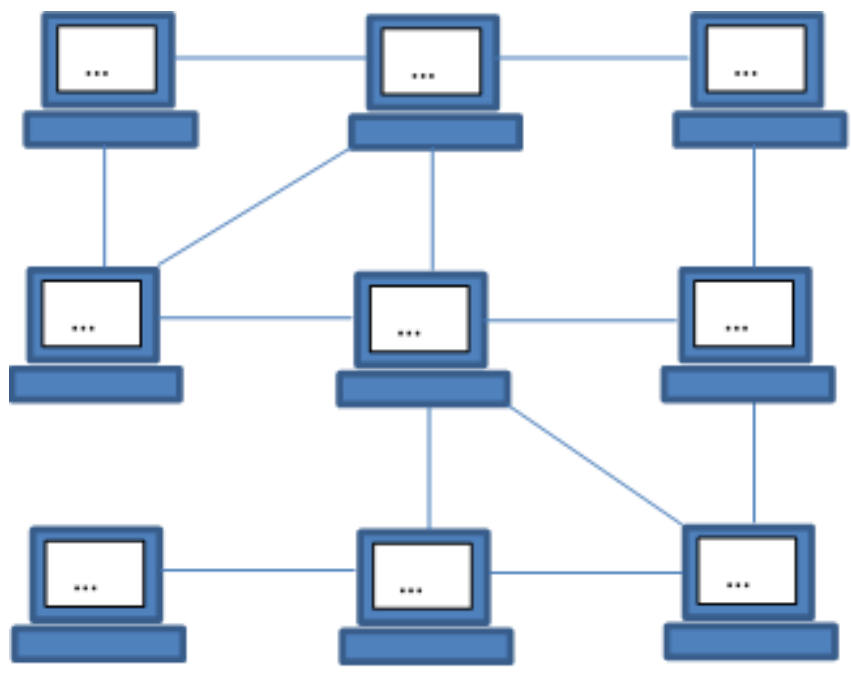

Fig. 3. Topología de red en Malla.
Una de las desventajas de esta topología de red es que debido a la necesidad de conexión de cada nodo entre sí, se requiere de una gran cantidad de cableado lo que la convierte en una topología muy costosa para el caso de redes cableadas. Así, su mayor implementación se presenta en redes inalámbricas [32].

Teniendo en cuenta las principales características descritas para las redes en malla, se hace evidente la gran aplicabilidad que tienen en ambientes rurales 0 agrestes en los cuales se pueden presentar diversas dificultades en algún nodo en particular, como destrucción de alguna estación (nodo) por parte de manos criminales, o por situaciones climatológicas, accidentes con animales, entre otros [33].

\section{PERSPECTIVA ECONÓMICA}

La inversión total que se requiere para el desarrollo de un proyecto en redes, suele separarse en dos tipos: el CAPEX y el OPEX [34].

EI CAPEX (capital de inversión) se refiere al despliegue en equipos los cuales con el tiempo se deprecian, como el equipamiento y las instalaciones (puntos de acceso, antenas, cableados, suiches, enrutadores, obras civiles y demás) y por su parte el OPEX (gastos de operaciones) representa los gastos mínimos de operación, como la administración y el mantenimiento de la red, gastos que no pueden ser amortizados (diseño, dotación de oficinas, servicios públicos y demás) [35]. 
Estos gastos ocasionan que la inversión para estos proyectos deba ser alta y esto es uno de los limitantes al acceso a Internet en países en desarrollo o ciertas áreas rurales de países desarrollados. Sin embargo, la tecnología WiBack al utilizar Redes en Malla Inalámbricas (Wireless Mesh Networks, WMN) las cuales son autoconfigurables y autogestionables reducen significativamente estos costos debido a la casi nula necesidad de mantenimiento [36], [37].

Uno de las principales dificultades que tienen que afrontar este tipo de redes es la provisión de energía eléctrica [20] ya que al tratarse de escenarios principalmente rurales no siempre se cuenta con un sistema de energía eléctrica eficiente. Para el caso colombiano, según datos del banco mundial, el $97 \%$ de la población contaba con servicio de energía eléctrica al finalizar el 2013 [38]; sin embargo, este porcentaje disminuye considerablemente en las zonas rurales. Una de las alternativas implementadas para la provisión de energía eléctrica a estas redes son los paneles solares, lo que corresponde a un tipo de energía que viene ganando relevancia en el país.

El presidente de la República de Colombia, anunció el 13 de mayo de 2014 la sanción de la Ley 1715 del 13 de mayo del 2014 [39] mediante la cual se busca motivar la producción de energía eléctrica a través de métodos y tecnologías limpias, buscando así eliminar la dependencia de una energía generada a partir del agua y del carbón. Con esta ley se busca además, la inclusión al sistema eléctrico colombiano a las zonas no interconectadas con lo cual se estaría solventando en parte, el problema del suministro energético para las redes WiBack.

\section{VENTAJAS Y DESVENTAJAS DE LA TECNOLOGÍA WIBACK}

La Tecnología WiBack, al utilizar una topología de red en malla, tiene la gran ventaja de que no requiere de un servidor central, además, el hecho de que los enrutadores WiBack se pueden instalar en cualquier construcción de altura considerable utilizada para otros propósitos, reduce el gasto en infraestructura ya que no se debe construir nuevos espacios de ubicación [40]. Al basarse en el estándar 802.21, puede hacer uso de las tecnologías inalámbricas existentes como WI-FI y WIMAX lo que implica que no se deben adquirir nuevos equipos disminuyendo los gastos. Así mismo, es importante anotar que implementa la tecnología de antenas MIMO, lo cual implica que se pueden manejar tasas de transmisión altas y se reduce la tasa de error aumentando la eficiencia espectral [41].

Otra gran ventaja es el bajo consumo de potencia requerida, lo cual lo hace ideal para trabajar por medio del uso de energías alternativas como los paneles solares [42]. Además, se garantiza una alta calidad de servicio con gran disponibilidad para tráfico de voz y datos sin sacrificar la distancia ya que se puede lograr igualmente el cubrimiento de grandes áreas a través de los enlaces de radio [43].

La principal desventaja que presenta esta tecnología es que al ser muy nueva, todavía se encuentra en desarrollo y no ha tenido una acogida a nivel internacional que asegure su supervivencia en el mercado [44].

\section{CONCLUSIONES}

Las tecnologías de la Información y la Comunicación (TIC), están cada vez más involucradas en los diversos aspectos de nuestras vidas: educación, economía, cultura, política, salud, entre otros, teniendo en cuenta que la forma como éstas influyen en cada uno de los aspectos mencionados es diferente.

Entre las diversas formas de TIC, es Internet la de mayor impacto en la sociedad actual, convirtiéndose en la principal herramienta de todo tipo de instituciones a lo largo del mundo, y así mismo, es considerada como agente vital para el desarrollo de una nación. Sin embargo, no todas las personas de una región dada pueden contar con los servicios de Internet, debido en la mayoría de los casos a situaciones económicas o geográficas desfavorables, incrementado así lo que se conoce como brecha digital.

Aunque en los últimos años, se ha logrado aumentar significativamente la inclusión digital en Colombia, la penetración de banda ancha sigue siendo baja en relación con otros países. Este aumento, se debe en gran parte a medidas del gobierno nacional como eliminar el gravamen del IVA de los computadores de gama baja, la posibilidad de adquisición de equipos de cómputo a través de las tarifas de servicios públicos y la reducción general precios de estos equipos por parte de los fabricantes.

Sin embargo, no todas las personas que poseen un equipo de cómputo, poseen conexión a Internet. Entre las principales causas que generan esta brecha digital se tienen principalmente: bajo poder adquisitivo de los ciudadanos, recursos limitados de las regiones y dificultades geográficas (especialmente en zonas rurales).

Como se mencionó antes, el gobierno nacional viene trabajando fuertemente en la inclusión digital a través de su proyecto "Vive Digital", pero especialmente en los temas relacionados a la parte económica y académica y poco se trata el tema de aquellos lugares que por su compleja ubicación geográfica no cuentan con este vital servicio.

El territorio colombiano rico en variedad de ecosistemas tales como cordilleras, valles, selvas, altiplanos, páramos y nevados, no posee la geografía idónea para el desarrollo de la infraestructura requerida para los sistemas de comunicaciones. Es por esta 
razón que se convierte en una tarea sumamente difícil proveer servicio de Internet a ciertas poblaciones que se encuentran en lugares alejados de los centros urbanos ya que esto supone grandes inversiones de dinero y posiblemente un impacto negativo en los diversos ecosistemas. Por lo tanto, y con la necesidad de vincular dichas regiones a la red de Internet, se requieren alternativas de conexión que permitan avanzar en su inclusión digital.

Ya en la comunidad europea y Estados Unidos, se viene investigando hace unos años en la posibilidad del despliegue de Internet por medio inalámbrico, utilizando las bandas de frecuencia que originalmente han sido destinadas para la radiodifusión de la televisión análoga en la porción UHF del espectro radioeléctrico [45].

La migración de la televisión analógica a televisión digital, asume la liberación de una gran franja de este espectro [46], frecuencias las cuales podrían ser utilizadas para la transmisión de la señal de Internet hacia aquellos lugares que debido a su compleja ubicación no cuentan con la infraestructura necesaria para la conectividad a la red.

Es así como en julio de 2011, el Instituto de Ingenierías Eléctrica y Electrónica (IEEE), estableció el estándar 802.22 para la Wireless Regional Area Network (WRAN) el cual utiliza los espectros blancos del espectro de frecuencias de la televisión analógica [47].

El objetivo de la tecnología WiBack no es convertirse en una alternativa a los operadores móviles sino proveer una infraestructura de transporte $y$ complementar las tecnologías inalámbricas existentes. WiBack soporta diferentes tipos de tecnologías inalámbricas gracias al uso del protocolo de la IEEE 802.21 [48].

Así pues, la inversión en equipamiento no es muy alta ya que se dispone de económicos equipos de tecnologías de uso masivo como WI-FI y WIMAX, los cuales gracias a su alta difusión pueden ser conseguidos muy fácilmente y a precios relativamente bajos. Los enrutadores WiBack pueden ser ubicados en torres existentes, edificios, tanques de agua, entre otros lo que reduce significativamente la inversión en infraestructura adicional, por lo tanto, el grueso de la inversión se requeriría solo para la etapa de diseño lo que garantiza la economía del despliegue de este tipo de proyectos.

Entre las diversas tecnologías que soporta WiBack se encuentran WI-FI y WPAN las cuales operan en las bandas de frecuencias que no requieren licenciamiento como las ISM (Industrial, Scientific and Medical) [49] lo que disminuye los costos que implica el Permiso del Uso del Espectro (PUE) según lo estipula el decreto 4392 de 2010 [50].
La tecnología WiBack puede ser utilizada para llevar Internet a países en desarrollo, zonas rurales en países desarrollados, como red de expansión en eventos de asistencia masiva o como red de despliegue rápido en eventos de catástrofes en cualquier región específica; incluso, esta tecnología puede ser utilizada para llevar Internet hacia islas ubicadas cerca de los litorales costeros [51].

Provee una disponibilidad comprobada de hasta unos cinco nueves lo que la convierte en una red que garantiza la calidad del servicio y a un muy bajo precio de montaje y operación.

\section{REFERENCIAS}

[1] O. Nottebohm; J. Manyica; J. Bughin; M. Chui; A. R. Syed and O. Ledezma. "En línea y en crecimiento: el impacto de internet en los países aspirantes" 2010. Online [octubre, 2013].

[2] M. Cimoli. "Las TIC para el crecimiento y la igualdad: renovando las estrategias de la sociedad de la información" Tercera Conferencia Ministerial sobre la Sociedad de la Información de América Latina y el Caribe, Noviembre, 2010.

[3] M. E. Andrade Zambrano; A. M. Holguín Moreira S. P. Zambrano Cevallos. "Impacto de las Tics en el desempeño de las Pymes en el Ecuador". Cantón Portoviejo, provincia de Manabí, diciembre, 2010.

[4] Ministerio de Tecnologías de la Información y las Comunicaciones de Colombia (MinTIC). "Vive Digital. Documento Vivo del Plan" Versión 1.0. Online [mayo, 2010].

[5] M. Guerra and V. Jordán. "Políticas públicas de Sociedad de la Información en América Latina: ¿una misma visión?" Pensamiento Iberoamericano 5, 2010.

[6] Fraunhofer Focus. "Introducing Fraunhofer's Wireless Backhaul Technology Wireless Backhaul Technlology". An overview of the concepts and the current status regarding our ongoing R\&D efforts. Online [mayo, 2014].

[7] S. J. N. Noutat; T. D. Ndié and C. Tangha. "Wireless Community Network Services: Opportunities and Challenges for DCs: Case of Rural Cameroon" eInfrastructure and e-Services for Developing Countries. Springer Berlin Heidelberg, 2013, pp. 308317.

[8] United Nations Conference on Trade and Development. "Trade and Development Report, 2010". United Nations New York and Geneva, 2010. Online [febrero, 2010].

[9] R. Raúl. "El papel de las TIC en el desarrollo" Vol. 19. Raul Katz, 2009.

[10] Departamento Administrativo Nacional de Estadística (DANE). "Encuesta Nacional de Calidad de Vida 2013". Cód.: DIE-020-PD-01-r5_V.2. Online [marzo, 2014].

[11] Ministerio de Tecnologías de la Información y las Comunicaciones de Colombia (MinTIC). "Boletín de seguimiento a metas de gobierno" Enero 2014. Online [febrero, 2014]. 
[12] TIC Digital. "En Colombia tendremos mayor conectividad y menor costo". La E-Magazine de la Tecnología, La Comunicación Digital y el Emprendimiento Empresarial del nuevo siglo. Online [marzo, 2014].

[13] Ministerio de Tecnologías de la Información y las Comunicaciones de Colombia (MinTIC). "Barreras que Impiden la Masificación de Internet". Online [mayo, 2014]

[14] Ministerio de Tecnologías de la Información y las Comunicaciones de Colombia (MinTIC). "Los computadores en Colombia son los más económicos de la región" Online [mayo, 2014].

[15]E. Sardi. "Cambios sociodemográficos en Colombia. Periodo Intercensal 1993 - 2005". Revista de la información básica. Online [enero, 2014].

[16] Fraunhofer Focus. "Wireless Bachaul Technology (WiBack)". Online [mayo, 2014].

[17]D. Corral. "Red alternativa de telecomunicaciones rurales en Ecuador" Máster en Redes de Telecomunicación para Países en Desarrollo. Tutor: Dr. Carlos Figuera Pozuelo. Escuela Técnica Superior de Ingeniería de Telecomunicación. Universidad Rey Juan Carlos. Madrid, España, 2010.

[18] C. Kobel; W. Baluja Garcia and J. Habermann. "A survey on Wireless Mesh Network applications in rural areas and emerging countries". Global Humanitarian Technology Conference (GHTC), IEEE, 2013.

[19]M. Kretschmer; P. Hasse; C. Niephaus; T. Horstmann and Jonas. "Connecting Mobile Phones via Carrier-Grade Meshed Wireless Back-Haul Networks". E-Infrastuctures and E-Services for Developing Countries. Springer Berlin Heidelberg, 2011. pp. 1-10.

[20] C. Niephaus and M. Kretschmer. "Towards an Energy Management Framework for Carrier-Grade Wireless Back-Haul Networks". Computer Communications and Networks (ICCCN), 2012 21st International Conference on. IEEE, 2012.

[21] M. Mathias; C. Niephaus; D. Henkel and G. Ghinea. "QoS-aware wireless back-haul network for rural areas with support for broadcast services in practice". Mobile Adhoc and Sensor Systems (MASS), 2011 IEEE 8th International Conference on. IEEE, 2011.

[22] A. Banchs; N. Bayer; D. Chieng; A. de la Oliva; B. Gloss; M. Kretschme and F. Zdarsky. "Carmen: Delivering carrier grade services over wireless mesh networks". Personal, Indoor and Mobile Radio Communications, PIMRC 2008. IEEE 19th International Symposium on. IEEE, 2008.

[23] P. Batroff; G. Ghinea; T. Horstmann; K. Jonas and J. Moedeker. "A Pilot of a QoS-Aware Wireless Back-Haul Network for Rural Areas". eInfrastructure and e-Services for Developing Countries. Springer Berlin Heidelberg, 2012. pp. 96105.

[24] IEEE. "IEEE standard for local and metropolitan area networks- part 21: Media independent handover" IEEE Std 802.21-2008, pp. c1 -301, jan. 2009.

[25] M. Kretschmer; C. Niephaus and G. Ghinea. "A Wireless Back-Haul Architecture Supporting Dynamic Broadcast and White Space Coexistence". Computer Communications and Networks (ICCCN), 2012 21st International Conference on. IEEE, 2012.

[26] A. de La Oliva; A. Banchs; I. Soto; T. Melia and A. Vidal. "An overview of IEEE 802.21: mediaindependent handover services". Wireless Communications, IEEE 15.4, 2008, pp. 96-103.

[27]D. Henkel; S. Englander; M. Kretschmer and C. Niephaus. "Connecting the unconnectedEconomic constraints and technical requirements towards a back-haul network for rural areas" GLOBECOM Workshops (GC Wkshps), IEEE, 2011.

[28] I. F. Akyildiz; X. Wang and W. Wang. "Wireless mesh networks: a survey". Computer networks 47.4, 2005, pp. 445-487.

[29] R. Draves; J. Padhye and B. Zill. "Routing in multiradio, multi-hop wireless mesh networks". Proceedings of the 10th annual international conference on Mobile computing and networking. ACM, 2004.

[30] R. Bruno; C. M. Conti and E. Gregori. "Mesh networks: commodity multihop ad hoc networks". Communications Magazine, IEEE 43.3, 2005, pp.123-131.

[31]J. Jun and M. L. Sichitiu. "The nominal capacity of wireless mesh networks". Wireless Communications, IEEE 10.5, 2003, pp. 8-14.

[32] W. S. Soh; Z. Antoniou and H. S. Hyong. "Improving restorability in radio access network" Global Telecommunications Conference, 2003. GLOBECOM'03. IEEE, vol. 6, 2003.

[33] G. Egeland and P. E. Engelstad. "The reliability and availability of wireless backhaul mesh networks" Wireless Communication Systems. 2008. ISWCS'08. IEEE International Symposium on. IEEE, 2008.

[34] S. M. Mishra; J. Hwang; D. Filippini; R. Moazzami; L. Subramanian and T. Du. "Economic analysis of networking technologies for rural developing regions" Internet and Network Economics. Springer Berlin Heidelberg, 2005, pp. 184-194.

[35]D. Soldani and S. Dixit. "Wireless relays for broadband access [radio communications series]". Communications Magazine, IEEE 46.3, 2008, pp.58-66.

[36] M. Kretschmer; P. Batroff; C. Niephaus and G. Ghinea. "Topology discovery and maintenance for heterogeneous wireless back-haul networks supporting unidirectional technologies". Communications (MICC), 2011 IEEE 10th Malaysia International Conference on. IEEE, 2011.

[37] G. Egeland and P. E. Engelstad. "The economy of redundancy in wireless multi-hop networks". Wireless Communications and Networking Conference, 2009, WCNC. IEEE, 2009.

[38] Banco Mundial. "Acceso a la electricidad (\% de población)". Online [diciembre, 2013]. 
[39] Ministerio de Minas y Energía, República de Colombia (MME). Gobierno nacional sanciona ley que incentiva el uso de energías renovables. Online [mayo, 2014].

[40] H. Viswanathan and S. Mukherjee. "Throughputrange tradeoff of wireless mesh backhaul networks". Selected Areas in Communications, IEEE Journal on 24.3, 2006, pp. 593-602.

[41] E. M. Mohamed; D. Kinoshita; K. Mitsunaga; Y. Higa and H. Furukawa."MIMO based wireless backhaul". Ultra-Modern Telecommunications and Control Systems and Workshops (ICUMT), 2010 International Congress on. IEEE, 2010.

[42] G. Fettweis and E. Zimmermann. "ICT energy consumption-trends and challenges". Proceedings of the 11th International Symposium on Wireless Personal Multimedia Communications, vol. 2, No 4, 2008.

[43] H. Y. Tung; K. F. Tsang; L. T. Lee and K. T. Ko. "QoS for mobile WiMAX networks: call admission control and bandwidth allocation". Consumer Communications and Networking Conference, 2008. CCNC 2008. $5^{\text {th }}$. IEEE, 2008.

[44]R. Nadiv and T. Naveh. "Wireless backhaul topologies: Analyzing backhaul topology strategies". Ceragon White Paper, 2010.

[45] M. Fitch; M. Nekovee; S. Kawade; K. Briggs and R. MacKenzie. "Wireless service provision in TV white space with cognitive radio technology: A telecom operator's perspective and experience". Communications Magazine, IEEE 49.3, 2011, pp.64-73.
[46] M. Nekovee. "Cognitive radio access to TV white spaces: Spectrum opportunities, commercial applications and remaining technology challenges". New Frontiers in Dynamic Spectrum, 2010 IEEE Symposium on. IEEE, 2010.

[47] IEEE. "IEEE standard for information technologytelecommunications and information exchange between systems wireless regional area networks (wran)-specific requirements part 22: Cognitive wireless ran medium access control (mac) and physical layer (phy) specifications: Policies and procedures for operation. 2014.

[48] T. Horstmann; M. Kretschmer; J. Modeker; C. Niephaus and S. Sauer. "Development framework for prototyping heterogeneous multi-radio wireless networks". Computer Communications and Networks (ICCCN), 2011 Proceedings of 20th International Conference on. IEEE, 2011.

[49] J. A. Park; S. K. Park; D. H. Kim; P. D. Cho and K. R. Cho. "Experiments on radio interference between wireless $L A N$ and other radio devices on a 2.4 GHz ISM band". Vehicular Technology Conference, 2003. VTC 2003-Spring. The 57th IEEE Semiannual, vol. 3. IEEE, 2003.

[50] Ministerio de Tecnologías de la Información y las Comunicaciones de Colombia (MinTIC). "Decreto 4392 de 2010" Online [Mayo, 2014].

[51]K. S. Zaidi; V. Jeoti and A. Awang. "Wireless backhaul for broadband communication over Sea". Communications (MICC), Malaysia International Conference on. IEEE, 2013. 